TRANSACTIONS OF THE

AMERICAN MATHEMATICAL SOCIETY

Volume 355, Number 3, Pages 1237-1250

S 0002-9947(02)03167-7

Article electronically published on October 17, 2002

\title{
ARE HAMILTONIAN FLOWS GEODESIC FLOWS?
}

\author{
CHRISTOPHER MCCORD, KENNETH R. MEYER, AND DANIEL OFFIN
}

\begin{abstract}
When a Hamiltonian system has a "Kinetic + Potential" structure, the resulting flow is locally a geodesic flow. But there may be singularities of the geodesic structure; so the local structure does not always imply that the flow is globally a geodesic flow. In order for a flow to be a geodesic flow, the underlying manifold must have the structure of a unit tangent bundle. We develop homological conditions for a manifold to have such a structure.

We apply these criteria to several classical examples: a particle in a potential well, the double spherical pendulum, the Kovalevskaya top, and the $N$-body problem. We show that the flow of the reduced planar $N$-body problem and the reduced spatial 3-body are never geodesic flows except when the angular momentum is zero and the energy is positive.
\end{abstract}

\section{INTRODUCTION}

Geodesic flows are always Hamiltonian. That is, given a Riemannian manifold $M$, the metric $G$ can be considered as a function on either the tangent bundle or the cotangent bundle $T^{*} M$. The cotangent bundle has a natural symplectic structure, and so the function $G$ considered as a Hamiltonian defines a Hamiltonian flow on $T^{*} M$. This flow is the same as the geodesic flow defined by the metric $G$ when it is transferred to the cotangent bundle [1].

In this paper we begin the study of the inverse problem [10] and ask when is a Hamiltonian flow a geodesic flow, or more generally a reparameterization of a geodesic flow. There are important classical results along these lines.

- A collection of holonomic constraints on a mechanical system defines a configuration manifold $M$ as a submanifold of some Euclidean space, and hence $M$ inherits a Riemannian structure. In the absence of any external forces, the mechanical systems evolve by the geodesic flow on $M$ [30. (A pea sliding on a surface without friction and under no external forces moves along a geodesic of the surface.)

- The flow of a classical mechanical system is locally a reparameterization of a geodesic flow. If the system has sufficient energy, its flow is globally a geodesic flow. A classical mechanical system has a Hamiltonian of the form $H=K+V$, where $V: M \rightarrow \mathbb{R}$ is a smooth potential function on the configuration manifold $M$ and $K$ is the kinetic energy. $K$ can be considered

Received by the editors January 2, 2002 and, in revised form, May 10, 2002.

2000 Mathematics Subject Classification. Primary 37N05, 34C27, 54H20.

Key words and phrases. Hamiltonian systems, geodesic flows, Jacobi metric, three-body problem.

This research was partially supported by grants from the Taft Foundation, the NSF and the NSERC.

(C)2002 American Mathematical Society 
as a Riemannian metric on $M$, and $H$ as a Hamiltonian on the symplectic manifold $T^{*} M$. The Jacobi metric is $G=(h-V)^{-1} K$, with $h$ a constant. It is a well-defined metric at those points of $M$ where $V<h$. The geodesic flow on $G=1$ is a reparameterization of the Hamiltonian flow on $H=h$ [1], 30]. (The flow of the spherical pendulum on an energy level sufficiently high that the bob can go over the top is a reparameterization of a geodesic flow on the unit sphere bundles of the 2-sphere.)

- Belbruno and Osipov [3, 4, [26] showed that the flow of the Kepler problem in $\mathbb{R}^{n}$ on an energy level $E$ is equivalent to a reparameterization of the geodesic flow on the unit tangent bundle of a manifold of constant curvature $-E$ with one point removed. Their theorem extends the work of Conley and Moser 24, who showed that the flow of the Kepler problem with negative energy is equivalent to the geodesic flow on the unit tangent bundle of the $n$-sphere with the north pole removed. Also see Milnor's survey article [23.

In 17 we gave some homological necessary conditions for a flow to admit a cross section. In this paper we shall in a similar manner give some necessary homological conditions for the flow to be a reparameterization of a geodesic flow. Our necessary conditions follow from the simple observation that such a flow lives on the unit tangent bundle of the base manifold, and this in turn places natural restrictions on the homology. Henceforth, a geodesic flow shall mean a geodesic flow or a

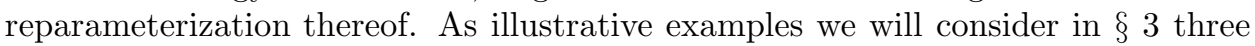
problems: a particle moving in a potential well; the double spherical pendulum; and a special case of the Kovalevskaya top. In these examples, we typically find that either there is enough energy for the system to be geodesic with respect to the Jacobi metric, or our criteria show that the flow on the energy level is not a geodesic flow.

In a series of papers [16, 17], 18, [19] we have computed the homology of the integral manifolds of the various versions of the three-body and $N$-body problem. In $\S$ 4, we apply our geodesic criteria to the reduced integral manifolds. We find that the flows on the reduced integral manifolds are almost never geodesic flows. That is, in all cases except that of zero angular momentum and positive energy, the integral manifolds of the planar $N$-body problem and of the spatial 3-body problem are not geodesic flows. In $\S$, we show that the flow of the planar $N$-body problem is a geodesic flow for zero angular momentum and positive energy.

\section{Necessary Conditions}

Given an odd-dimensional manifold $P$ with a flow, a necessary condition for the flow to be a geodesic flow is that the underlying manifold $P$ must be a unit tangent bundle. That is, if $\operatorname{dim}(P)=2 n-1$, there must be an $n$-dimensional manifold $M$ such that $P$ is the unit tangent bundle $T_{0} M$ of $M$. If $P$ is the unit tangent bundle of some manifold, this will put restrictions on the homology of $P$, which can be viewed as necessary conditions for $P$ to admit a geodesic flow. We formulate and apply these conditions in this section, and give their proofs in Section 6 .

Unless otherwise indicated, $H_{*}(P)$ will denote the homology of $P$ with integer coefficients. When the homology groups $H_{k}(P)$ are finitely generated, they have a unique decomposition $H_{k}(P)=F_{k}(P) \oplus T_{k}(P)$ where $F_{k}(P)$ is torsion-free and $T_{k}(P)$ is a finite group. As a free group, $F_{k}(P)=\mathbb{Z}^{\beta_{k}}$, where $\beta_{k}$ (sometimes written 
$\beta_{k}(P)$ for emphasis) is known as the $k$ th Betti number of $P$. The Betti numbers $\beta_{k}(P)$ and the torsion groups $T_{k}(P)$ will provide the necessary information about $P$.

There will be various cases to consider, depending on whether or not the base manifold is compact or non-compact, and whether it is orientable or non-orientable. Before generating necessary conditions for each, we need to be able to distinguish these four cases, using only information from the space $P$ and its homology. The first result shows that this is possible. With only one possible ambiguity, we can distinguish each possible combination of orientability and compactness from the homology of $P$.

Theorem 2.1. Suppose $P$ is a connected manifold of dimension $2 n-1$. Then the following are necessary conditions for $P$ to be the unit tangent bundle $T_{0} M$ of an $n$-manifold $M$ :

- If $M$ is compact and orientable, then $\beta_{2 n-1}(P)=1$ and $T_{n-1}(P)$ is cyclic or trivial.

- If $M$ is compact and non-orientable, then $\beta_{2 n-1}(P)=1$ and $T_{n-1}(P)$ has order 4 .

- If $M$ is non-compact and orientable, then $\beta_{2 n-1}(P)=0$ and $T_{n-1}(P)$ is trivial.

- If $M$ is non-compact and non-orientable, then $\beta_{2 n-1}(P)=0$ and $T_{n-1}(P)$ has order 2.

Moreover, in all cases, $P$ must be orientable as a manifold, and $H_{2 n-1}(P)$ and $H_{2 n-2}(P)$ must be torsion-free.

Note that the four cases are distinct, except when $P$ is compact with $\beta_{2 n-1}(P)=$ 1 and $T_{n-1}(P)=\mathbb{Z}_{4}$. In that exceptional case, the orientability of the base manifold $M$ cannot be determined. When the orientability of the base manifold can be guaranteed (i.e., when $P$ is compact and $T_{n-1}(P) \neq \mathbb{Z}_{4}$, or when $P$ is non-compact and $T_{n-1}(P)=0$ ), there are additional necessary conditions that can be applied. If the orientability of the base manifold cannot be guaranteed, then the conditions that follow are not valid. Analogous necessary conditions can be formulated in the non-orientable case by using homology with $\mathbb{Z}_{2}$ coefficients. However, since most naturally occurring examples are orientable, we will simplify the presentation and describe only the orientable case here.

Theorem 2.2. Suppose $P$ is a connected orientable non-compact manifold of dimension $2 n-1$, with $T_{k}(P)=0$ for $k=n-1,2 n-2$ and $2 n-1$. Then necessary conditions for $P$ to be the unit tangent bundle of some orientable $n$-manifold $M$ are:

(1) $\beta_{0}(P)=1$;

(2) $\beta_{2 n-1}(P)=0$;

(3) $\beta_{k}(P)=\beta_{k+n-1}(P)$ for all $1 \leq k \leq n-2$; and

(4) $\beta_{n-1}(P)=\beta_{2 n-2}(P)+1$.

In particular, there is one Betti number that must be non-zero:

Corollary 2.1. If $P$ is a non-compact connected orientable manifold of dimension $2 n-1$, a necessary condition for $P$ to be the unit tangent bundle of some orientable $n$-manifold $M$ is $\beta_{n-1}(P) \neq 0$. 
When $P$ is compact, the situation is rather different. Corollary 2.1 no longer holds, but there are other, stronger conditions that do apply. To simplify the formulation of the general result, the low-dimensional case $n=2$ will be considered separately in Theorem 3.1 .

Theorem 2.3. If $P$ is a compact connected orientable manifold of dimension $2 n-1$ with $n>2$ and $T_{n-1}(P)$ cyclic or trivial, then necessary conditions for $P$ to be the unit tangent bundle of some orientable $n$-manifold $M$ are:

(1) $\beta_{2 n-1}(P)=1$;

(2) $\beta_{k}(P)=\beta_{k+n-1}(P)$ for all $2 \leq k \leq n-2$;

(3) $0 \leq \beta_{n-1}(P)-\beta_{2 n-2}(P)=\beta_{n}(P)-\beta_{1}(P) \leq 1$.

(4) If the torsion subgroup $T_{n-1}(P)$ is non-trivial, then

$$
\begin{aligned}
& \quad\left|\sum_{i=0}^{n-2}(-1)^{i} \beta_{i}+(-1)^{n}\left(1-\beta_{2 n-2}\right)\right|=\left|T_{n-1}(P)\right| . \\
& \text { If } T_{n-1}(P)=0 \text {, then } \\
& \qquad\left|\sum_{i=0}^{n-2}(-1)^{i} \beta_{i}+(-1)^{n}\left(1-\beta_{2 n-2}\right)\right|+\beta_{n}(P)-\beta_{1}(P)=1 .
\end{aligned}
$$

The last condition is easily computed, and will be the most important condition in applications. For emphasis, we reformulate the most important case:

Corollary 2.2. If $P$ is a compact, connected orientable manifold of dimension $2 n-1, n>2$, and $T_{n-1}(P)$ trivial, then a necessary condition for $P$ to be the unit tangent bundle of some orientable $n$-manifold is

$$
\left|\sum_{i=0}^{n-2}(-1)^{i} \beta_{i}+(-1)^{n}\left(1-\beta_{2 n-2}\right)\right|=1+\beta_{1}(P)-\beta_{n}(P) .
$$

\section{ILlustrative Examples}

The results of Section 2, in particular Corollaries 2.1 and 2.2 can be applied to several simple classical systems.

Particle in a potential well. Consider a classical Hamiltonian $H=K(p)+V(q)$ where $(q, p) \in \mathbb{R}^{n} \times \mathbb{R}^{n}, n \geq 2$. Assume the kinetic energy $K(p)$ is a positive definite quadratic form in the momenta, $p$; that the potential energy $V: \mathbb{R}^{n} \rightarrow \mathbb{R}$ has a unique critical point at $q=0$ which is a non-degenerate minimum; and that $V \rightarrow \infty$ as $\|q\| \rightarrow \infty$. Then Morse theory [22] implies that $H^{-1}(h), h>V(0)$, is a $(2 n-1)$-sphere. Lemma 3.1 will show that no sphere satisfies the condition of Corollary 2.2. Thus the flow on an energy surface is not geodesic.

Lemma 3.1. The $(2 n-1)$-dimensional sphere is not homeomorphic to the unit tangent bundle of an $n$-manifold.

Proof. The $(2 n-1)$-sphere has torsion-free homology with Betti numbers $\beta_{0}=$ $\beta_{2 n-1}=1, \beta_{i}=0,2 \leq i \leq 2 n-2$. The spheres satisfy all of the hypotheses of Corollary 2.2 so to admit a unit tangent structure, $S^{2 n-1}$ must satisfy the condition in (1). Inserting the Betti numbers of a sphere, we obtain the condition $1+(-1)^{n}=1$, which is clearly never satisfied. 
TABLE 1. The table of Betti numbers for the double pendulum

\begin{tabular}{|c|c|c|c|c|c|c|c|c|}
\hline$\beta_{p}$ & 0 & 1 & 2 & 3 & 4 & 5 & 6 & 7 \\
\hline$-5<h<-3$ & 1 & 0 & 0 & 0 & 0 & 0 & 0 & 1 \\
\hline$-3<h<3$ & 1 & 0 & 1 & 0 & 0 & 1 & 0 & 1 \\
\hline $3<h<5$ & 1 & 0 & 2 & 0 & 0 & 2 & 0 & 1 \\
\hline $5<h$ & 1 & 0 & 2 & 0 & 0 & 2 & 0 & 1 \\
\hline
\end{tabular}

Double spherical pendulum. By the double spherical pendulum we mean the mechanical system consisting of two coupled spherical pendula in a constant gravitational field. To be specific, let the position vectors of the two pendula be $q_{1}$ and $q_{2}$ with fixed lengths $l_{1}=2$ and $l_{2}=1$ and with masses $m_{1}=m_{2}=1$. Assume the acceleration due to gravity is $g=1$. The configuration space is $S^{2} \times S^{2}$, the product of a sphere of radius 2 and a sphere of radius 1 . The energy of the system is

$$
E(q, \dot{q})=\frac{1}{2}\left\|\dot{q}_{1}\right\|+\frac{1}{2}\left\|\dot{q}_{2}\right\|+\left(2 q_{1}+q_{2}\right) \cdot k,
$$

where $k=(0,0,1)$. For any energy level $h>-5$, the energy level set

$$
M(h)=\left\{(q, \dot{q}) \in T\left(S^{2} \times S^{2}\right) \mid E(q, \dot{q})=h\right\}
$$

projects onto $C(h)=\left\{q \in S^{2} \times S^{2} \mid V(q) \leq h / 2\right\}$. For any $q \in C(h)$, the preimage of the projection $\phi: M(h) \rightarrow C(h)$ is $\phi^{-1}=\left\{p \in \mathbb{R}^{3} \mid p^{2}=h-2 V(q)\right\}$. This is a 3 -sphere if $V(q)<h / 2$, and a point if $V(q)=h / 2$. That is, since $\{V(q)=h / 2\}$ is precisely the boundary of $C(h)$ in $S^{2} \times S^{2}$, we can describe $M(h)$ as a 3 -sphere bundle over $C(h)$, with the spheres collapsing to points on the zero velocity set $\partial C(h)$.

From this description, we can detect the energy levels at which bifurcations in the manifolds $M(h)$ occur, and can compute the homology of $M(h)$ in each parameter interval. Clearly the topology of $M(h)$ can only change when the topology of $C(h)$ changes. As a sub-level set of the potential function, this can only occur at the bifurcation values of the potential function. The potential has four critical points with critical values -5 (both pendula down), -3 (long pendulum down, short pendulum up), +3 (long pendulum up, short pendulum down), and 5 (both pendula up). Thus, there are four distinct energy ranges for the structure of the manifold $M(h)$. The Betti numbers of $M(h)$ for each range are listed in Table 1 .

Note that when $h>5$ there are no restrictions on the configuration of the pendulum, and for each $q \in S^{2} \times S^{2}$, the fiber $\phi^{-1}(q)$ is a sphere in $T_{q}\left(S^{2} \times S^{2}\right)$. That is, $M(h)$ is (up to a rescaling) the unit tangent bundle of $S^{2} \times S^{2}$. In fact, the flow is the geodesic flow of the Jacobi metric. In all the other cases, the torsion subgroup $T_{3}(M(h))=0$. So Corollary 2.2 would require the identity

$$
\left|\beta_{0}-\beta_{1}+\beta_{2}+1-\beta_{6}\right|=1+\beta_{1}-\beta_{4} .
$$

In all cases, $\beta_{0}=1$ and $\beta_{1}=\beta_{4}=\beta_{6}=0$; so the identity simplifies to $\left|2+\beta_{2}\right|=1$. Since $\beta_{2}$ is non-negative, this equality is clearly never satisfied. Thus, for $h<5$, the flow is not a geodesic flow.

Kovalevskaya top. In his classic paper, Iacob [12] classifies the integral manifolds of a Kovalevskaya top. This three-degree-of-freedom problem has a symmetry and the integrals of energy and angular momentum. Fixing energy equal to $h$ and 
TABle 2. The table of Betti numbers for the Kovalevskaya top

\begin{tabular}{|c|c|c|c|c|}
\hline$\beta_{p}$ & 0 & 1 & 2 & 3 \\
\hline$S^{3}$ & 1 & 0 & 0 & 1 \\
\hline$S^{1} \times S^{2}$ & 1 & 1 & 1 & 1 \\
\hline$\left(S^{1} \times S^{2}\right) \#\left(S^{1} \times S^{2}\right)$ & 1 & 2 & 2 & 1 \\
\hline$R P^{3}$ & 1 & 0 & 0 & 1 \\
\hline
\end{tabular}

the magnitude of angular momentum equal to $p \neq 0$ defines a four-dimensional invariant subset of the phase space $I_{h, p} . I_{h, p}$ is invariant under $\mathrm{SO}_{2}$ action that leaves the angular momentum integral fixed. The quotient space $\tilde{I}_{h, p}=I_{h, p} / S O_{2}$ is in general a three-dimensional manifold. A collection of curves (the bifurcation set) divides the $(h, p)$-parameter space into four open domains where $\tilde{I}_{h, p}$ is of constant topological type. The four types are $S^{3}, S^{1} \times S^{2},\left(S^{1} \times S^{2}\right) \#\left(S^{1} \times S^{2}\right)$ (the connected sum), and $R P^{3}$ (real projective 3 -space). Table 2 lists the Betti numbers of these four topological types.

The unit circle bundle of $S^{2}$ is topologically $R P^{3}$. This is the case where there is sufficient energy for the Jacobi metric to be globally defined, and the flow of the top is a geodesic flow. In the other three cases, the torsion group $T_{1}=0$. So if the space is to be the unit tangent bundle of a surface, that surface must be orientable. Theorem 3.1 below will show that the only 3 -manifold that can be the unit tangent bundle of a compact, connected orientable surface is the 3 -torus $T^{3}$. The Betti number table shows that none of the remaining three manifolds is homeomorphic to $T^{3}$; so none of these can admit a geodesic structure. That is, the flow is a geodesic flow if and only if $\tilde{I}_{h, p}=R P^{3}$.

There is a vast literature on the motion of a rigid body, and many special cases have the same or similar types of integral manifolds - see for example [9], 13], 25] and the references therein.

Theorem 3.1. If $P$ is a compact connected orientable 3-manifold, then for $P$ to be the unit tangent bundle of an orientable surface, either the homology of $P$ must be that of the three-dimensional torus,

$$
H_{k}(P)= \begin{cases}\mathbb{Z}^{3}, & k=1,2, \\ \mathbb{Z}, & k=0,3, \\ 0, & k>3,\end{cases}
$$

or there must be a $g>1$ such that

$$
H_{k}(P)= \begin{cases}\mathbb{Z}^{2 g}, & k=2, \\ \mathbb{Z}^{2 g} \oplus \mathbb{Z}_{2-2 g}, & k=1, \\ \mathbb{Z}, & k=0,3, \\ 0, & k>3 .\end{cases}
$$

The proof is given in $\S 6.3$. Note that the theorem shows that there is only one compact 3-manifold with torsion-free homology which can be the unit tangent bundle of an orientable surface: the 3-torus, which is the unit tangent bundle of the 2-torus. 


\section{Geodesic Flows in the $N$-Body Problem}

The $N$-body problem refers to the motion of $N$ point masses under their mutual gravitational attraction. When the masses move in $\mathbb{R}^{3}$, the problem is referred to as the spatial $N$-body problem; when they are all restricted to a single plane, it is known as the planar $N$-body problem. In the $N$-body problem, the integral manifolds are the level sets of center of mass, linear momentum, angular momentum $\vec{c}$ and energy $h$. We denote the integral manifolds for the planar problem by $\mathfrak{m}(c, h)$ and the integral manifolds for the spatial problem by $\mathfrak{M}(c, h)$ (where $c=|\vec{c}|)$. The topology of these manifolds varies as $\vec{c}$ and $h$ vary. When $c \neq 0$, the topology of the integral manifolds depends only on the quantity $\nu=-h c^{2}$.

There are also rotational symmetries. In the planar problem, there is a natural $\mathrm{SO}_{2}$ symmetry. In the spatial problem, the cases of zero and non-zero angular momentum are distinct. With non-zero angular momentum, the angular momentum vector $\vec{c}$ creates a preferred direction, and the only symmetry is the $\mathrm{SO}_{2}$ symmetry of rotations about the angular momentum vector. For zero angular momentum, there is a full $\mathrm{SO}_{3}$ symmetry. In each case, the quotient space of the integral manifold is known as the reduced integral manifold, and represents the lowest-dimensional set on which the dynamics of the $N$-body problem is displayed. The reduced integral manifolds are denoted by $\mathfrak{M}_{R}(c, h)$ and $\mathfrak{m}_{R}(c, h)$, and have dimensions

$$
\begin{aligned}
\operatorname{dim}\left(\mathfrak{m}_{R}(c, h)\right) & =4 N-7, \\
\operatorname{dim}\left(\mathfrak{M}_{R}(c, h)\right) & = \begin{cases}6 N-11, & c \neq 0, \\
6 N-13, & c=0\end{cases}
\end{aligned}
$$

When the action is free, the reduced spaces are manifolds. The planar action is always free, as is the $\mathrm{SO}_{2}$ action in the spatial problem with non-zero angular momentum. But in the spatial problem with zero angular momentum, those configurations with all position and momentum vectors parallel have $S^{1}$ isotropy, while all other configurations have trivial isotropy. The presence of two different isotropy groups means that the reduced space is not a manifold. We will exclude this case from consideration.

In all other cases, the reduced integral manifolds are all odd-dimensional manifolds, while the integral manifolds themselves are all even-dimensional. So it is on the reduced manifolds that we might look for geodesic structures. Combining the topological and homological results of [6], [7], [16], [19] with the results of this paper, we can determine whether or not the $N$-body flow on the reduced integral manifolds is a geodesic flow in the following cases: (i) the planar $N$-body problem for all $N, c$ and $h$, (ii) the spatial 3-body problem for non-zero $c$ and all $h$, (iii) the spatial $N$-body problem with $h c^{2}$ positive.

The two remaining cases are the spatial $N$-body problem with $N \geq 4$, negative energy and non-zero angular momentum, and the spatial $N$-body problem with zero angular momentum. In both cases, we conjecture that the integral manifolds do not admit a geodesic structure. But there is at present no topological or homological description of these manifolds sufficiently detailed to allow the question to be decided. In all of the other cases, the results are as follows:

Theorem 4.1. In the planar $N$-body problem, the flow on the reduced integral manifold $\mathfrak{m}_{R}(c, h)$ is a geodesic flow if and only if $c=0$ and $h \geq 0$. 
In the spatial $N$-body problem with non-negative energy and non-zero angular momentum, the flow on the reduced integral manifold $\mathfrak{M}_{R}(c, h)$ is not a geodesic flow.

In the spatial 3-body problem with $h<0$ and $c \neq 0$, the flow on the reduced integral manifold $\mathfrak{M}_{R}(c, h)$ is not a geodesic flow.

Proof. With the exception of the planar problem with $c=0$ and $h \geq 0$, the results are all negative. All of the negative results are obtained by applying Corollary 2.1. The relevant homology group is $H_{2 N-4}\left(\mathfrak{m}_{R}(c, h)\right)$ in the planar problem; $H_{3 N-7}\left(\mathfrak{M}_{R}(0, h)\right)$ in the spatial problem with zero angular momentum; and $H_{3 N-6}\left(\mathfrak{M}_{R}(c, h)\right)$ in the spatial problem with non-zero angular momentum.

We will analyze the various cases by considering the different combinations of angular momentum (zero vs. non-zero) and energy (negative vs. non-negative) for both the spatial and planar problems.

Case $c=0, h \geq 0$ : In the planar problem, the $\mathrm{SO}_{2}$ action is free, and we shall show in Proposition 5.1 that the flow on $\mathfrak{m}_{R}(0, h)$ is a classical system. Since $h \geq 0$, the Jacobi metric is globally defined, and so the flow is geodesic.

Case $c=0, h<0$ : In the planar problem, Smale [27] showed that $\mathfrak{m}_{R}(0, h) \cong$ $\left(\mathbb{C} P^{N-2} \backslash \Delta\right) \times \mathbb{R}^{2 N-3}$, where $\Delta$ denotes the collision set. The homology of $\mathfrak{m}_{R}(0, h)$ is the same as that of $\mathbb{C} P^{N-2} \backslash \Delta$. As an open (2N-4)-manifold, it has no homology in dimension $2 N-4$, violating Corollary 2.1

Case $c \neq 0, h \geq 0$ : In the planar problem, the same result of Smale 27] applies: $\mathfrak{m}_{R}(0, h) \cong\left(\mathbb{C} P^{N-2} \backslash \Delta\right) \times \mathbb{R}^{2 N-3}$. In the spatial case, Cabral and McCord [7] show that the non-zero Betti numbers of $\mathfrak{M}_{R}(c, h)$ are strictly increasing, which violates the requirement $\beta_{k}=\beta_{k+n-1}$ of Theorem 2.2

Case $c \neq 0, h<0$ : In this range, there are no homology calculations for $\mathfrak{M}_{R}(c, h)$ for four or more bodies. For the 3-body problem, the cohomology groups are computed in [19, 1 In the spatial 3-body problem, it is $\beta_{3}$ that must be non-zero. But an inspection of the tables in [19] or [17] shows that $\beta_{3}=0$ for all energy levels.

In the planar $N$-body problem, we need to show that $\beta_{2 N-4}=0$. Applying the homology formula of [16, Theorem 1.3] to dimension $2 N-4$, we obtain

$$
\begin{aligned}
H_{2 N-4}\left(\mathfrak{m}_{R}(c, h)\right) & \cong H_{2 N-4}\left(\mathfrak{r}_{R}(\nu)\right) \oplus H_{-1}\left(\mathfrak{r}_{R}(\nu), \mathfrak{b}_{R}(\nu)\right) \\
& =H_{2 N-4}\left(\mathfrak{r}_{R}(\nu)\right),
\end{aligned}
$$

where $\mathfrak{r}_{R}(\nu)$ and $\mathfrak{r}_{R}(\nu)$ are superlevel- and level-sets of the potential function $V$ on the reduced configuration space $\mathbb{C} P^{N-2} \backslash \Delta$. That is,

$$
\mathfrak{r}_{R}(\nu)=\left\{q \in \mathbb{C} P^{N-2} \backslash \Delta \mid V(q) \geq \sqrt{\nu}\right\} \quad \text { and } \quad \mathfrak{b}_{R}(\nu)=V^{-1}(\sqrt{\nu}) .
$$

At a regular value, $\mathfrak{b}_{R}(\nu)$ has a tubular neighborhood in $\mathfrak{r}_{R}(\nu)$, and

$$
\mathfrak{r}_{R}(\nu) \simeq \mathfrak{r}_{R}(\nu) \backslash \mathfrak{b}_{R}(\nu) .
$$

Thus, $\mathfrak{r}_{R}(\nu)$ has the homology of a non-compact $(2 N-4)$-manifold, and, by Proposition 6.3

$$
H_{2 N-4}\left(\mathfrak{m}_{R}(\nu)\right) \cong H_{2 N-4}\left(\mathfrak{r}_{R}(\nu)\right) \cong 0
$$

\footnotetext{
${ }^{1}$ The table in [19] contains a mistake. The cohomology groups for positive energy are given incorrectly, and are corrected in [17].
} 


\section{Zero Angular Momentum}

As we have seen, the homology considerations are useful in identifying cases in which the reduced manifold does not have a geodesic structure. In contrast, there is one case when the flow of the $N$-body problem on the reduced space is a geodesic flow.

The following observations are implicit in 2], [14, and we include them to complete our discussion of the $N$-body problem. Refer to [1, 14 for background and additional details. A classical Hamiltonian system with symmetry is a Hamiltonian of the form $H=K+U$ where $H: T^{*} M \rightarrow \mathbb{R}, K_{q}\left(p_{q}\right)=\frac{1}{2}\left\|p_{q}\right\|_{q}^{2}$ is the kinetic energy, $U(q)$ is the potential energy, and additionally there is a Lie group action $G \times M \rightarrow M$ which leaves $U$ invariant and whose lift to the cotangent bundle is a symplectic action on $T^{*} M$ which leaves $H$ invariant. Let $\mathfrak{g}^{*}$ be the dual of the Lie algebra of $G$, and let $\langle\alpha, \beta\rangle=\alpha(\beta)$ when $\alpha \in \mathfrak{g}^{*}$ and $\beta \in \mathfrak{g}$.

The equivariant momentum map $\mathbf{J}: T^{*} M \rightarrow \mathfrak{g}^{*}$ is given by

$$
\left\langle\mathbf{J}\left(p_{q}\right), \xi\right\rangle=\left\langle p_{q}, \xi_{M}(q)\right\rangle
$$

where $\xi_{M}$ denotes the infinitesimal generator of $\xi$ on $M$. Noether's theorem asserts that $\mathbf{J}$ is an integral of the motion.

Let $\mu \in \mathfrak{g}^{*}$ be a regular value, $G_{\mu}=\{g \in G: g \cdot \mu=\mu\}, Q_{\mu}=\mathbf{J}^{-1}(\mu) / G_{\mu}$. In this situation the Meyer [20], Marsden-Weinstein [15] reduction theorem states that if the $G$-action is free and proper, then $Q_{\mu}$ is a smooth symplectic manifold and the flow defined by $H$ drops naturally to a Hamiltonian flow on $Q_{\mu}$. However, in general $Q_{\mu}$ is not a cotangent bundle and the reduced Hamiltonian is not the sum of kinetic energy plus potential energy. The next Proposition states that in case the momentum is zero, however, the reduced space is always a cotangent bundle.

Proposition 5.1. Let $\mu=0$ be a regular value and let the $G$-action on $M$ be free and proper, so that the quotient space $M / G$ is a smooth manifold. Then $Q_{0}=$ $\mathbf{J}^{-1}(0) / G$ is the cotangent bundle of $M / G$, and the reduced Hamiltonian system is a sum of a kinetic energy and a potential term. That is, if $\mu=0$, the reduced system is a classical system.

Remark. Since in this case the system is a classical system, the Jacobi metric is locally defined on $H=h$. The Jacobi metric will be globally defined provided $M \subset U^{-1}((-\infty, h])$.

Proof. There are several ways to prove this. The simplest observation is that when $\mu=0$ the amended potential is just the potential on the quotient space and from the equivariance everything drops down to the quotient space nicely — see the discussion in 14 for background.

For a direct approach, first observe that, by the equivariance, $G_{0}=G$. We will describe the reduced space $Q_{0}=\mathbf{J}^{-1}(0) / G$, by showing how to project along certain directions in the fiber to get the reduction to the zero momentum set. Define the following subspaces for a point $q \in M$ :

$$
\begin{aligned}
& W_{q}=\left\{\xi_{M}(q) \in T_{q} M: \xi_{M} \text { is the infinitesimal generator of } \xi \in \mathfrak{g}\right\}, \\
& H_{q}=W_{q}^{0}=\left\{p_{q} \in T_{q}^{*} M:\left\langle p_{q}, w\right\rangle=0, w \in W_{q}\right\} \\
& V_{q}=H_{q}^{\perp}=\left\{a_{q} \in T_{q}^{*} M: K_{q}\left(a_{q}, p_{q}\right)=0, \forall p_{q} \in H_{q}\right\} .
\end{aligned}
$$


$W_{q}$ is tangent to the $G$-orbit at $q, H_{q}$ is the annihilator of $W_{q}$, and $V_{q}$ is the $K$ orthogonal complement of $H_{q}$. Thus, $T_{q}^{*}=H_{q} \oplus V_{q}$. The key observation is that the horizontal space $H_{q}$ can be identified with the zero momentum level over $q \in M$.

By the equivarance $U$ is constant on $G$ orbits, and so we can define the reduced potential energy function $\tilde{U}: M / G \rightarrow \mathbb{R}$ by $\tilde{U}([q])=U(q)$.

Since we have separated out the zero momentum level $H_{q}$ of the fiber over $q \in M$, we now can project along the orthogonal complement $V_{q}$ to get the reduced space. However, as noted above, the vertical space $V_{q}$ is tangent to the group orbits at every point. Therefore, we can identify $\bigcup_{q} H_{q}$ with $T^{*}(M / G)$. Define $\tilde{K}$ as the restriction of the metric $K$ to the subspace $H_{q}$. If $p_{q} \in J^{-1}(0)$, then $0=\left\langle J\left(p_{q}\right), \mathfrak{g}\right\rangle=\left\langle p_{q}, W_{q}\right\rangle$ or $p_{q} \in W_{q}^{0}=H_{q}$; so if $p_{q} \in J^{-1}(0)$, then $K\left(p_{q}\right)=\tilde{K}\left(p_{q}\right)$. Thus, the Hamiltonian on the reduced space is $\tilde{H}=\tilde{K}+\tilde{U}$.

\section{Homology of Tangent Bundles}

This section develops the proofs of the results presented in $\S 2$ To develop necessary conditions for a manifold to have the structure of a unit tangent bundle, we start with such a structure, and work out its homological consequences. To understand the homology of $T_{0} M$, we will need to consider two features: compactness and orientability.

Proposition 6.1. If $M$ is a manifold, and $T_{0} M$ is its unit tangent bundle, then $M$ is compact if and only if $T_{0} M$ is compact.

Proposition 6.2. For any manifold $M, T_{0} M$ is an orientable manifold.

Proof. For any manifold $M$, its tangent bundle $T M$ is orientable. The sphere bundle is a codimension 1 subbundle that separates the tangent bundle; so it too is orientable.

That is, $T_{0} M$ is orientable, even when $M$ is not. However, the orientability of $M$ is tied to a different notion of orientability of $T_{0} M$ - that of $T_{0} M$ as a bundle over $M$. In fact, the requirement that $p: T_{0} M \rightarrow M$ be orientable is one way of defining the orientability of $M$. This is equivalent to requiring that $\pi_{1}(M)$ acts trivially on the homology of the fiber $H_{*}\left(S^{n-1}\right)$.

To investigate the homology of manifolds, these two properties of compactness and orientability are fundamental. Of course, it is the combination of compactness and orientability that guarantees duality. More useful for our purposes are the following properties [8]:

Proposition 6.3. If $M$ is an n-manifold, and $H_{*}(M)$ is the homology of $M$ with integer coefficients, then

(1) $H_{p}(M)=0$ for $p>n$.

(2) $H_{n}(M) \cong \begin{cases}\mathbb{Z} & \text { if } M \text { is compact and orientable, } \\ 0 & \text { otherwise. }\end{cases}$

(3) The torsion subgroup of $H_{n-1}(M)$ is

$$
T_{n-1}(M) \cong\left\{\begin{array}{cl}
\mathbb{Z}_{2} & \text { if } M \text { is compact and non-orientable }, \\
0 & \text { otherwise. }
\end{array}\right.
$$

Note that those parts of Theorem 2.1 which do not depend on the orientability or compactness of the base manifold (i.e., that $T_{0} M$ is always orientable, and that 
$H_{2 n-2}\left(T_{0} M\right)$ and $H_{2 n-1}\left(T_{0} M\right)$ are always torsion-free) follow immediately from these results. All of the other results of Section 2 will depend on the Gysin sequence [11], [29]. Given a sphere bundle $S^{n-1} \rightarrow E \rightarrow B$, there is an exact sequence

$$
\ldots \rightarrow H_{k-n+1}(B ; \mathcal{Z}) \rightarrow H_{k}(E) \rightarrow H_{k}(B) \rightarrow H_{k-n}(B ; \mathcal{Z}) \rightarrow \ldots,
$$

where $H_{*}(B ; \mathcal{Z})$ indicates the homology of $B$ with twisted coefficients. If $\pi_{1}(B)$ acts trivially on $H_{n-1}\left(S^{n-1}\right)$, then $H_{*}(B ; \mathcal{Z})=H_{*}(B)$; but if the action is nonorientable, $H_{*}(B ; \mathcal{Z})$ may be very difficult to compute. We will consider the two cases separately.

6.1. Non-orientable bundles. If the bundle is non-orientable, we cannot give as complete a description, but there are a few useful pieces of information that can be extracted.

First, if $M$ is a non-orientable manifold, then Proposition 6.3 guarantees that $H_{n}(M)=0$ and $H_{n-1}(M)=F_{n-1}(M) \oplus T_{n-1}(M)$, with $F_{n-1}(M)$ free abelian and $T_{n-1}$ equal to $\mathbb{Z}_{2}$ if $M$ is compact and trivial if it is not. The other useful result is that the homology with twisted coefficients has $H_{0}(M ; \mathcal{Z}) \cong \mathbb{Z}_{2}[29]$.

Since $H_{k}(M)=0$ for all $k<0$ and all $k \geq n$, there are no non-trivial boundary maps in the Gysin sequence, and for all $k$ we have

$$
0 \rightarrow H_{k-n+1}(M ; \mathcal{Z}) \rightarrow H_{k}\left(T_{0} M\right) \rightarrow H_{k}(M) \rightarrow 0 .
$$

In particular, in dimension $n-1$ we have

$$
0 \rightarrow \mathbb{Z}_{2} \rightarrow H_{n-1}\left(T_{0} M\right) \rightarrow F_{n-1}(M) \oplus T_{n-1}(M) \rightarrow 0 ;
$$

so the torsion subgroup of $H_{n-1}\left(T_{0} M\right)$ satisfies

$$
0 \rightarrow \mathbb{Z}_{2} \rightarrow T_{n-1}\left(T_{0} M\right) \rightarrow T_{n-1}(M) \rightarrow 0 .
$$

That is, the torsion group is $\mathbb{Z}_{2}$ if $M$ is non-compact, and has order four if $M$ is compact.

6.2. Orientable bundles. For an orientable manifold $M$, the Gysin sequence of the unit tangent bundle simplifies to

$$
\ldots \rightarrow H_{k-n+1}(M) \rightarrow H_{k}\left(T_{0} M\right) \rightarrow H_{k}(M) \rightarrow H_{k-n}(M) \rightarrow \ldots
$$

The boundary map $H_{k}(M) \rightarrow H_{k-n}(M)$ can only be non-trivial if $H_{k}(M)$ and $H_{k-n}(M)$ are non-trivial. From Proposition 6.3, we see that this can only occur when $k=n$ and $M$ is compact.

The Gysin sequence thus splits into the following sequences:

$$
0 \rightarrow H_{k}\left(T_{0} M\right) \rightarrow H_{k}(M) \rightarrow 0
$$

for $0 \leq k \leq n-2$,

$$
0 \rightarrow H_{1}(M) \rightarrow H_{n}\left(T_{0} M\right) \rightarrow H_{n}(M) \stackrel{\stackrel{\partial}{\rightarrow}}{\rightarrow} H_{0}(M) \rightarrow H_{n-1}\left(T_{0} M\right) \rightarrow H_{n-1}(M) \rightarrow 0,
$$

and

$$
0 \rightarrow H_{k-n+1}(M) \rightarrow H_{k}\left(T_{0} M\right) \rightarrow 0
$$

for $n+1 \leq k \leq 2 n-1$.

We can replace all of the groups $H_{k}(M)$ in these sequences, and obtain a set of relations between the various homology groups of $T_{0} M$. We consider the cases when $M$ is compact and non-compact separately. 
First, suppose $M$ is non-compact. Then $H_{n}(M)=0$, and the sequence simplifies to

$$
0 \rightarrow H_{k}\left(T_{0} M\right) \rightarrow H_{k}(M) \rightarrow 0
$$

for $0 \leq k \leq n-2$,

$$
0 \rightarrow \mathbb{Z} \rightarrow H_{n-1}\left(T_{0} M\right) \rightarrow H_{n-1}(M) \rightarrow 0,
$$

and

$$
0 \rightarrow H_{k-n+1}(M) \rightarrow H_{k}\left(T_{0} M\right) \rightarrow 0
$$

for $n \leq k \leq 2 n-1$. Combining these, we obtain the following relations between the homology groups of $T_{0} M$ :

- $H_{0}\left(T_{0} M\right)=\mathbb{Z}$

- $H_{2 n-1}\left(T_{0} M\right)=0$,

- $H_{k}\left(T_{0} M\right) \cong H_{k+n-1}\left(T_{0} M\right)$ for $1 \leq k \leq n-2$,

- $0 \rightarrow \mathbb{Z} \rightarrow H_{n-1}\left(T_{0} M\right) \rightarrow H_{2 n-2}\left(T_{0} M\right) \rightarrow 0$.

Since $H_{2 n-2}\left(T_{0} M\right)$ is torsion-free, it follows from the exact sequence that $H_{n-1}\left(T_{0} M\right)$ is likewise. Thus the non-compact, orientable condition of Theorem 6.2 follows from these relations, as does Theorem 2.2.

Now, suppose $M$ is compact. The only difference lies in the boundary map $H_{n}(M) \stackrel{\partial}{\rightarrow} H_{0}(M)$. If $M$ is compact, connected and orientable, then $H_{n}(M) \cong$ $H_{0}(M) \cong \mathbb{Z}$. The boundary map is a homomorphism $\mathbb{Z} \rightarrow \mathbb{Z}$, which can also be thought of as multiplication by an integer. In fact, that integer is simply the Euler characteristic of $M, \chi(M)=\sum_{i=0}^{n} \beta_{i}(M)$ [11]. The non-trivial portion of the Gysin sequence becomes

$$
0 \rightarrow H_{1}(M) \rightarrow H_{n}\left(T_{0} M\right) \rightarrow \mathbb{Z} \stackrel{\chi(M)}{\longrightarrow} \mathbb{Z} \rightarrow H_{n-1}\left(T_{0} M\right) \rightarrow H_{n-1}(M) \rightarrow 0 .
$$

Combining this with $H_{k}\left(T_{0} M\right) \cong H_{k}(M)$ for $0 \leq k \leq n-2$ and $H_{k-n+1}(M) \cong$ $H_{k}\left(T_{0} M\right)$ for $n+1 \leq k \leq 2 n-1$, we have

- $H_{0}\left(T_{0} M\right) \cong H_{2 n-1}\left(T_{0} M\right) \cong \mathbb{Z}$,

- $H_{k}\left(T_{0} M\right) \cong H_{k+n-1}\left(T_{0} M\right)$ for $2 \leq k \leq n-2$, and

- there is an exact sequence

$$
0 \rightarrow H_{1}\left(T_{0} M\right) \rightarrow H_{n}\left(T_{0} M\right) \rightarrow \mathbb{Z} \stackrel{\chi(M)}{\longrightarrow} \mathbb{Z} \rightarrow H_{n-1}\left(T_{0} M\right) \rightarrow H_{2 n-2}\left(T_{0} M\right) \rightarrow 0 .
$$

Since $H_{2 n-2}\left(T_{0} M\right)$ is torsion-free, the torsion subgroup of $H_{n-1}\left(T_{0} M\right)$ is the cokernel $\mathbb{Z} / \chi(M) \mathbb{Z}$ of $\chi(M): \mathbb{Z} \rightarrow \mathbb{Z}$. That is, $T_{n-1}\left(T_{0} M\right)$ must be cyclic, with $\left|T_{n-1}\left(T_{0} M\right)\right|=|\chi(M)|$. This completes the proof of Theorem 2.1 The Betti number relations of Theorem 2.3 also follow immediately. The only element of the theorem requiring further discussion is the formula

$$
\sum_{i=0}^{n-2}(-1)^{i} \beta_{i}\left(T_{0} M\right)+(-1)^{n}\left(1-\beta_{2 n-2}\left(T_{0} M\right)\right)=\left|T_{n-1}\left(T_{0} M\right)\right| .
$$

Having established that $\left|T_{n-1}\left(T_{0} M\right)\right|=|\chi(M)|$, it remains only to show that

$$
\chi(M)=\sum_{i=0}^{n-2}(-1)^{i} \beta_{i}\left(T_{0} M\right)+(-1)^{n}\left(1-\beta_{2 n-2}\left(T_{0} M\right)\right) .
$$


The identities $H_{k}\left(T_{0} M\right) \cong H_{k}(M)$ for $0 \leq k \leq n-2$ and $H_{k-n+1}(M) \cong H_{k}\left(T_{0} M\right)$ for $n+1 \leq k \leq 2 n-1$ imply that

$$
\beta_{i}(M)= \begin{cases}\beta_{i}\left(T_{0} M\right), & 0 \leq i \leq n-2, \\ \beta_{i+n-1}\left(T_{0} M\right), & 2 \leq i \leq n\end{cases}
$$

Inserting these, and observing that $\beta_{n}(M)=\beta_{2 n-1}\left(T_{0} M\right)=1$, provides the result.

6.3. Tangent bundles of surfaces. We close with the proof of Theorem 3.1.

Proof. From the classification of surfaces, we know that a compact orientable surface is characterized by its genus. The genus $g$ surface has homology

$$
H_{k}\left(S_{g}\right)= \begin{cases}\mathbb{Z}^{2 g}, & k=1, \\ \mathbb{Z}, & k=0,2, \\ 0, & k>2,\end{cases}
$$

and Euler characteristic $\chi\left(S_{g}\right)=2-2 g$. The unit tangent bundle, as a compact, connected orientable 3 -manifold, has $H_{0}\left(T_{0} S_{g}\right) \cong H_{3}\left(T_{0} S_{g}\right) \cong \mathbb{Z}$. To determine $H_{1}$ and $H_{2}$, we turn to the Gysin sequence

$$
0 \rightarrow H_{1}\left(S_{g}\right) \rightarrow H_{2}\left(T_{0} S_{g}\right) \rightarrow H_{2}\left(S_{g}\right) \rightarrow H_{0}\left(S_{g}\right) \rightarrow H_{1}\left(T_{0} S_{g}\right) \rightarrow H_{1}\left(S_{g}\right) \rightarrow 0 .
$$

Inserting the known values for $H_{*}\left(S_{g}\right)$, this becomes

$$
0 \rightarrow \mathbb{Z}^{2 g} \rightarrow H_{2}\left(T_{0} S_{g}\right) \rightarrow \mathbb{Z} \stackrel{2-2 g}{\longrightarrow} \mathbb{Z} \rightarrow H_{1}\left(T_{0} S_{g}\right) \rightarrow \mathbb{Z}^{2 g} \rightarrow 0 .
$$

When $g=1$ (i.e., when the surface is the torus), the map $2-2 g=0$ and $H_{2}\left(T_{0} S_{g}\right) \cong H_{1}\left(T_{0} S_{g}\right) \cong \mathbb{Z}^{3}$. In all other cases, multiplication by $2-2 g$ produces the torsion subgroup $\mathbb{Z}_{2-2 g}$ in $H_{1}\left(T_{0} S_{g}\right)$.

\section{REFERENCES}

1. R. Abraham and J. Marsden, Foundations of Mechanics, Benjamin Cummings, London, 1978. MR 81e:58025

2. V. I. Arnold, Dynamical Systems III, Encyclopaedia of Math.Sci., vol. 3, Springer-Verlag, New York, 1988. MR 88m:58043

3. E. A. Belbruno, Two-body motion under the inverse square central force and equivalent geodesic flows, Celest. Mech. 15(4) (1977) 467-476. MR 57:4239

4. - Regularization and geodesic flows, Classical Mechanics and Dynamical Systems, (Eds. R. Devaney and Z. Nitecki), Lecture Notes in Pure and Applied Mathematics, Marcel Dekker, New York, 1981, 1-11. MR 82k:58002

5. G. D. Birkhoff, Dynamical Systems, Amer. Math. Soc., Providence, RI, 1927.

6. H. Cabral, On the Integral Manifolds of the N-Body Problem, Invent. Math. 20 (1973) 59 72. MR 49:9870

7. H. Cabral and C. K. McCord, Topology of the integral manifolds of the spatial $N$-Body Problem with positive energy, J. Dyn. Diff. Eqs. 14 (2002), 259-293.

8. A. Dold, Lectures on algebraic topology, Springer-Verlag, New York: 1972. MR 54:3685

9. H. R. Dullin, Die Energieflächen des Kowalewskaja-Kreisels, Verlag Mainz, Wissenschaftsverlag, Aachen, 1994. MR 97f:70009

10. C. W. Groetsch, Inverse Problems in the Mathematical Sciences, Vieweg, Wiesbaden, 1993. MR 94m:00008

11. D. Husemoller, Fibre bundles, Graduate Texts in Mathematics, No. 20. Springer-Verlag, New York-Heidelberg, 1975. MR 51:6805

12. A. Iacob, Invariant manifolds in the motion of a rigid body about a fixed point, Rev. Roum. Math. Pures Appl., XVI(10), 1971, 1-25. MR 46:2937

13. M. P. Kharlamov, Topological analysis of integrable problems of rigid body dynamics (Russian), Leningrad. Univ., Leningrad, 1988. MR 89i:58036

14. J. E. Marsden, Lectures on Mechanics, London Math. Soc. Lecture Note Ser. 174, Cambridge University Press, Cambridge, 1992. MR 93f:58078 
15. J. E. Marsden and A. Weinstein, Reduction of symplectic manifolds with symmetries, Rep. Math. Phys., 5 (1974), 121-130. MR 53:6633

16. C. K. McCord, On the homology of the integral manifolds in the planar N-body problem, Ergodic Theory \& Dynamical Systems 21 (2001) 861 - 883.

17. C. K. McCord and K. R. Meyer, Cross Sections in the Three-Body Problem, J. Dyn. Diff. Eqs. 12 (2000), 247-271. MR 2001k:70011

18. Integral manifolds of the restricted three-body problem, Ergodic Theory $\&$ Dynamical Systems 21 (2001) 885 - 914.

19. C. K. McCord, K. R. Meyer and Q. Wang, The integral manifolds of the three-body problem, Mem. Amer. Math. Soc., 628 (1998) 1-91. MR 98i:70004

20. K. R. Meyer, Symmetries and integrals in mechanics, Dynamical Systems, M. Peixoto (ed.), Academic Press, New York, 1973. MR 48:9760

21. K. R. Meyer and G. R. Hall, Introduction to Hamiltonian Dynamical Systems and the N-body Problem, Springer-Verlag, New York, 1992. MR 93b:70002

22. J. Milnor, Morse Theory, Princeton Univ. Press, Princeton, NJ, 1963. MR 29:634

23. $ـ$ On the geometry of the Kepler problem, Amer. Math. Monthly, 90 (1983) 353-365. MR 84i:70008

24. J. K. Moser, Regularization of Kepler's problem and the averaging method on a manifold, Comm. Pure Appl. Math. XXIII (1970) 609-635. MR 42:4824

25. A. A. Oshemkov, Fomenko invariants for the main integrable cases of the rigid body motion equations, Topological Classification of Integrable Systems, (Ed. A. T. Fomenko), Amer. Math. Soc., 1991, 67-146. MR 93b:58057

26. Yu Osipov, The Kepler problem and geodesic flows in spaces of constant curvature, Celest. Mech., 16, 1977, 191-208. MR 58:18598

27. S. Smale, Topology and Mechanics, II. Invent. Math., 11 (1970) 45 - 64. MR 47:9671

28. A. Wintner, The Analytical Foundations of Celestial Mechanics, Princeton Univ. Press, 1941. MR 3:215b

29. G. W. Whitehead, Elements of homotopy theory, Graduate Texts in Mathematics, 61. Springer-Verlag, New York-Berlin, 1978. MR 80b:55001

30. E. T. Whittaker, A Treatise on the Analytic Dynamics of Particles and Rigid Bodies with an Introduction to the Problem of Three Bodies, 4th ed., Cambridge Univ. Press, 1937.

University of Cincinnati, Cincinnati, Ohio 45221-0025

E-mail address: CHRIS.MCCORD@UC.EDU

University of Cincinnati, Cincinnati, Ohio 45221-0025

E-mail address: KEN.MEYER@UC.EDU

Queen's University, Kingston, Ontario K7L 4V1, Canada

E-mail address: OFFIND@MAST. QUEENSU.CA 